\title{
Comparison of the Pharmacokinetic Profiles of a Standardized Extract of Centella asiatica and A Mixture of Madecassoside and Asiaticoside in Rats
}

\section{다)(1)}

\author{
Authors \\ Phisit Khemawoot ${ }^{1,2}$, Patcharaporn Hengjumrut ${ }^{1}$, Tosapol Anukunwithaya ${ }^{1}$, Leng Chee Chang ${ }^{3}$, \\ Supakit Wongwiwatthananukit ${ }^{3}$, Mayuree H. Tantisira ${ }^{4}$
}

\section{Affiliations}

1 Department of Pharmacology and Physiology, Faculty of Pharmaceutical Sciences, Chulalongkorn University, Bangkok, Thailand

2 Preclinical Pharmacokinetics and Interspecies Scaling for Drug Development Research Unit, Chulalongkorn University, Bangkok, Thailand

3 Daniel K. Inouye College of Pharmacy, University of Hawai' ${ }^{\prime}$, Hilo, HI, USA

4 Faculty of Pharmaceutical Sciences, Burapha University, Chonburi, Thailand

Key words

Centella asiatica, Apiaceae, ECa 233, madecassoside, asiaticoside, pharmacokinetics

$\begin{array}{lr}\text { received } & 16.11 .2017 \\ \text { revised } & 14.03 .2018 \\ \text { accepted } & 19.03 .2018\end{array}$

Bibliography

DOI https://doi.org/10.1055/a-0600-9750

Planta Med Int Open 2018; 5: e39-e47

(c) Georg Thieme Verlag KG Stuttgart · New York

ISSN 2509-9264

Correspondence

Phisit Khemawoot, PhD

Department of Pharmacology and Physiology

Faculty of Pharmaceutical Sciences

Chulalongkorn University, Phyathai Road

Bangkok 10330

Thailand

Tel.: +66/2/218 8343, Fax: +66/2/218 8340

phisit.k@chula.ac.th
Aupporting information is available online at

http://www.thieme-connect.de/products

\begin{abstract}
Centella asiatica is a tropical plant commonly used as an herbal remedy in traditional medicines in many countries. In an attempt to establish an herbal extract with well-defined characteristics, a standardized extract of C. Asiatica, ECa 233, was developed. This extract contains at least $80 \%$ triterpenoid glycosides with the major constituents madecassoside and asiaticoside at a ratio of $1.5 \pm 0.5: 1$. In the present study, comparative pharmacokinetics of ECa 233 with its mixture of madecassoside and asiaticoside were conducted in rats. Following intravenous or oral administration of the test compounds, blood, tissues, urine, and feces were collected for the determination of madecassoside, asiaticoside, and their metabolite levels using liquid chromatography tandem mass spectrometry. Plasma levels of madecassoside and asiaticoside in the ECa 233-treated group were found to be higher than their respective counterparts in the mixture. Madecassoside and asiaticoside in both test formulae appeared to be widely distributed in several organs, and more than $50 \%$ of the administered doses were recovered as madecassic acid and asiatic acid in the feces within 24 to $48 \mathrm{~h}$. The results clearly demonstrated the pharmacokinetic advantage of a standardized extract of $C$. Asiatica, ECa 233, compared with a mixture of madecassoside and asiaticoside at an equivalent amount. Other minor constituents that naturally exist in ECa 233 appeared to positively modulate the pharmacokinetics of its major constituents, resulting in relatively better pharmacokinetic profiles than those from a mixture of pure compounds.
\end{abstract}

\begin{tabular}{|c|c|c|c|}
\hline $\mathrm{Abt}$ & ons & $\mathrm{AUC}_{0-24}$ & area under the curve from time zero to $24 \mathrm{~h}$ \\
\hline ALT & alanine aminotransferase & $A \cup C_{0-i n f}$ & area under the curve from time zero to infinity \\
\hline AST & aspartate aminotransferase & $\mathrm{C}_{\max }$ & maximal plasma concentration \\
\hline
\end{tabular}




$\begin{array}{ll}\mathrm{Cl}_{\text {app }} & \text { apparent clearance } \\ \text { ECa 233 } & \text { standardized extract of Centella asiatica } \\ \text { IS } & \text { internal standard } \\ \text { IV } & \text { intravenous } \\ \text { Kp } & \text { tissue to plasma ratio } \\ \text { MRT } & \text { mean resident time } \\ \text { NSS } & \text { normal saline solution } \\ \text { PO } & \text { per oral } \\ \mathrm{T}_{1 / 2} & \text { elimination half-life } \\ \mathrm{T}_{\max } & \text { time to reach maximal plasma concentration } \\ \mathrm{V}_{\text {app }} & \text { apparent volume of distribution } \\ \text { XlogP } & \text { partition coefficient }\end{array}$

\section{Introduction}

Traditional medicine serves as an alternative choice for disease prevention and treatment for people in developing countries. Centella asiatica (L.) Urb., a tropical herb belonging to the Apiaceae family, is commonly used in alternative and traditional medicines in Asia [1-3]. C. asiatica from different sources showed significant variations in the quality and quantity of its bioactive compounds, leading to unpredictable pharmacological activities. Using a strictly controlled method, we developed a standardized extract of $C$. asiatica, ECa 233 [4, 5], which was defined as a white to off-white extracted powder of $C$. asiatica that contained triterpenoid glycosides, namely, madecassoside and asiaticoside, in an amount of at least $80 \%$ and the ratio between them was maintained at 1.5 \pm 0.5:1 (• Fig. 1). ECa 233 exhibited pharmacological profiles that corresponded to the traditional use of $C$. asiatica in humans; that is, wound healing properties, neuroprotective properties, and memory-enhancing effects [6-9]. In parallel with the favorable pharmacological profiles, ECa 233 demonstrated minimal toxicity in both acute and subchronic toxicity tests in rodents [10].

Recently, our laboratory reported pharmacokinetic profiles following single and multiple oral dosings of ECa 233 in rodents $[11,12]$. This standardized extract showed dose linearity within a pharmacologically effective dose range of $50-200 \mathrm{mg} / \mathrm{kg} P O$ in male Wistar rats. Madecassoside and asiaticoside, in a clear solu-

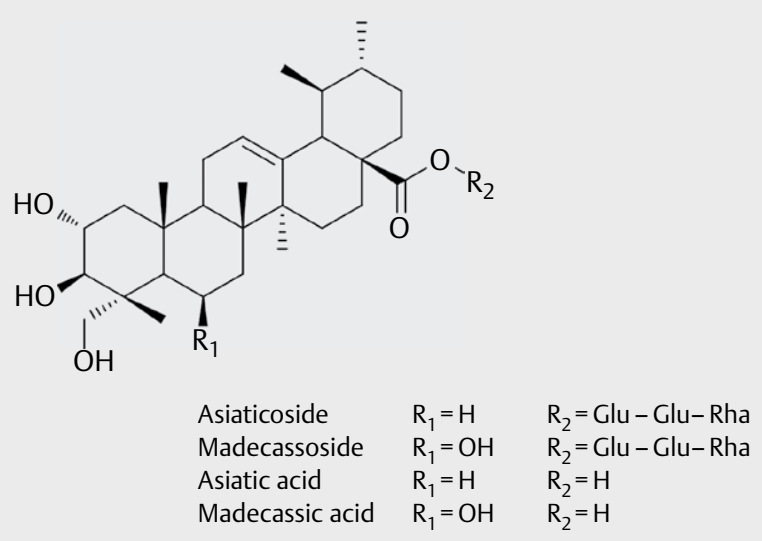

Fig. 1 Chemical structures of triterpenes from C. asiatica (Glu: glucose, Rha: rhamnose). tion of ECa 233, rapidly reached the maximum concentration in plasma within $1 \mathrm{~h}$ following oral dosing and were prominently detected in most pharmacologically relevant organs (e. g., skin and brain). Negligible amounts of the proposed active metabolites, madecassic and asiatic acids, were detected in rat plasma, and, unexpectedly, we found a bidirectional interconversion between madecassoside and asiaticoside [13]. Both triterpenoid glycosides were mainly excreted via the hepatobiliary system, and were extensively biotransformed into their respective triterpenic acids, madecassic and asiatic acids, likely by normal flora in the gastrointestinal tract $[14,15]$. Interestingly, in relation to the pharmacokinetics of its respective single compounds, an increased exposure of the two triterpenoid glycosides present in ECa 233 was observed. Questions on whether other minor constituents in ECa 233 may underlie increased exposure to its major bioactive constituents, madecassocide and asiaticoside, therefore, emerged and required clarification. To obtain more insight into the results previously observed, we compared the pharmacokinetic behavior of ECa 233 compared it with that exhibited by a mixture of madecassoside and asiaticoside at equivalent amounts. The pharmacologically active dose of ECa 233 at $100 \mathrm{mg} / \mathrm{kg}$ PO, containing $51 \%$ madecassoside and $38 \%$ asiaticoside, was administered to male Wistar rats. Accordingly, a mixture of $51 \mathrm{mg} / \mathrm{kg}$ madecassoside and $38 \mathrm{mg} / \mathrm{kg}$ asiaticoside was prepared for the comparative pharmacokinetic study. In addition, a study of the pharmacokinetics following intravenous administration of ECa 233 at $10 \mathrm{mg} / \mathrm{kg}$ and a mixture of madecassoside and asiaticoside at an equivalent amount was also conducted. In addition to madecassoside and asiaticoside, other minor constituents are also present in ECa 233 and their impact on the major constituents remains elusive. Information obtained from this study could illustrate the significance of interactions among the different chemical constituents that are present in ECa 233.

\section{Results}

All rats had a normal physical appearance both before and after receiving ECa 233 or the mixture of madecassoside and asiaticoside. There were no significant changes in the creatinine level, reflecting normal kidney function after dosing for all test groups at $24 \mathrm{~h}$. Similarly, stable AST and ALT levels were observed after dosing in all of the test groups. There were no significant alterations in liver markers for any of the animals tested, either via IV or PO routes of administration ( $\triangleright$ Table 1 ).

Significant differences in the plasma concentration-time profiles between madecassoside and asiaticoside present in ECa 233 and their respective counterparts at equivalent doses in the mixture groups were demonstrated ( $\triangleright$ Fig. 2, 3). After IV dosing, rats in the ECa 233 group exhibited significantly higher plasma concentrations of madecassoside and asiaticoside compared with their respective counterparts in the mixture group. Similarly, oral administration of ECa 233 could prolong exposure of both madecassoside and asiaticoside compared to the results observed with the mixtures. Determination of pharmacokinetic parameters from the plasma concentration-time curves by non-compartmental analysis is presented in > Table $\mathbf{2}, \mathbf{3}$. In comparison with the pharmacokinetic profiles of the mixture of madecassoside and asiaticoside, an 


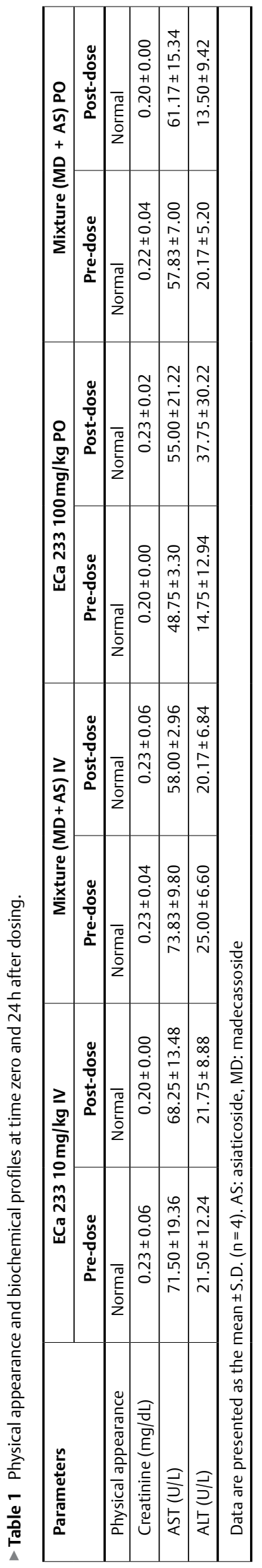

increase of approximately $400 \%$ of the madecassoside area under the curve (AUC) and an increase of $50 \%$ of the asiaticoside AUC were detected following IV dosing of ECa 233. Similarly, a significant increase of both the madecassoside and asiaticoside AUCs were also observed following oral dosing with ECa 233. The MRT of both madecassoside and asiaticoside following dosing with ECa 233 showed a tendency to prolong in comparison to these components in the mixture.

The tissue to plasma values (Kp) of madecassoside and asiaticoside following IV dosing of ECa 233 or the mixture at an equivalent dose are presented in $\mathbf{F i g}$. 4. Madecassoside and asiaticoside could be concentrated in and distributed to several organs, including the skin and brain, which were target sites of pharmacological actions. The accumulation of madecassoside and asiaticoside in the spleen was observed from 1 to $4 \mathrm{~h}$ after dosing with ECa 233 or the mixture. Interestingly, the Kp values of both madecassoside and asiaticoside increased significantly from 1 to $4 \mathrm{~h}$ following IV dosing of both test formulae. ECa 233 showed higher Kp values of both madecassoside and asiaticoside in some organs at 1 and $2 \mathrm{~h}$ compared with the mixture.

Negligible amounts of unchanged madecassoside and asiaticoside were found in excreta following the IV administration of all test formulae ( $\triangleright$ Table 4 ). After dosing for $24-48 \mathrm{~h}$, it was likely that most of the administered madecassoside and asiaticoside were extensively biotransformed into madecassic and asiatic acids, before being excreted in feces. There were no significant differences in the concentrations of madecassic and asiatic acids in feces between the ECa 233 and mixture groups.

The compounds of the ECa 233 extract were analyzed using HPLC ESI-QTOF-MS/MS and two standards (madecassoside and asiaticoside). The first major peak of the mass chromatogram was detected at $7.0 \mathrm{~min}$ with a molecular weight of 997.4995 daltons (Da). This molecular weight was deduced to be a sodium adduct $[\mathrm{M}+\mathrm{Na}]^{+}$of madecassoside. The second major peak detected at 7.95 min exhibited a molecular weight of 981.5034 Da (• Fig. 5). This molecular weight was deduced to be a sodium-adduct $[\mathrm{M}+\mathrm{Na}]^{+}$of asiaticoside. These two major peaks were confirmed using standards that had the same retention time eluted with the same solvent systems and molecular weight. MS/MS fragmentation data provided further evidence for the presence of madecassoside and asiaticoside in the ECa 233 extract. In addition, a minor peak at 7.38 min with a molecular weight of 981.5047 Da was detected. This compound was considered to likely be a sodium adduct $[\mathrm{M}+\mathrm{Na}]^{+}$of centellasaponins, which had an exact molecular weight corresponding to 958.51 Da plus sodium 22.99 Da. However, the specific type of centellasaponins (A, C, D) remained undetermined due to the lack of commercially available centellasaponins. Overall, by matching retention times, mass spectra of sodium ion adducted molecular ions, and fragmentation patterns of madecassoside and asiaticoside, it was evident that the minor components of ECa 233 extracts contain a centellasaponin.

Additionally, two small peaks of minor components at 13.0 and 15.0 min were found. These signals were confirmed to be madecassic and asiatic acids, consistent with their retention times and molecular weights compared with analytical standards. These triterpenic acids accounted for less than $1.0 \%$ of ECa 233 using quantitative analysis with a triple quadrupole LC-MS/MS system. 

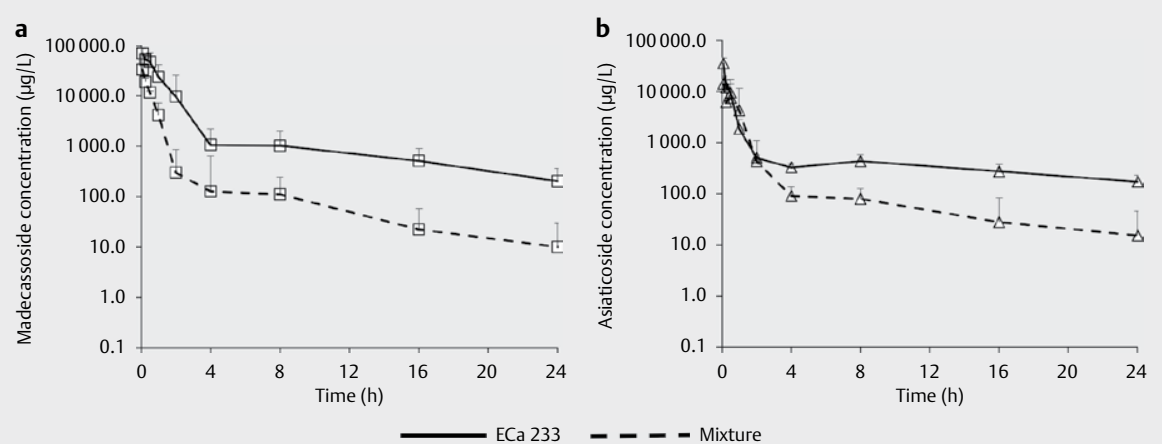

Fig. 2 Plasma concentration-time profiles of madecassoside (a) and asiaticoside (b) following intravenous administration of ECa 233 (10 mg/kg) and a mixture of madecassoside and asiaticoside at an equivalent dose. Data are presented as the mean \pm S.D. $(n=4) ;{ }^{*} p<0.05$ for ECa 233 vs. mixture.
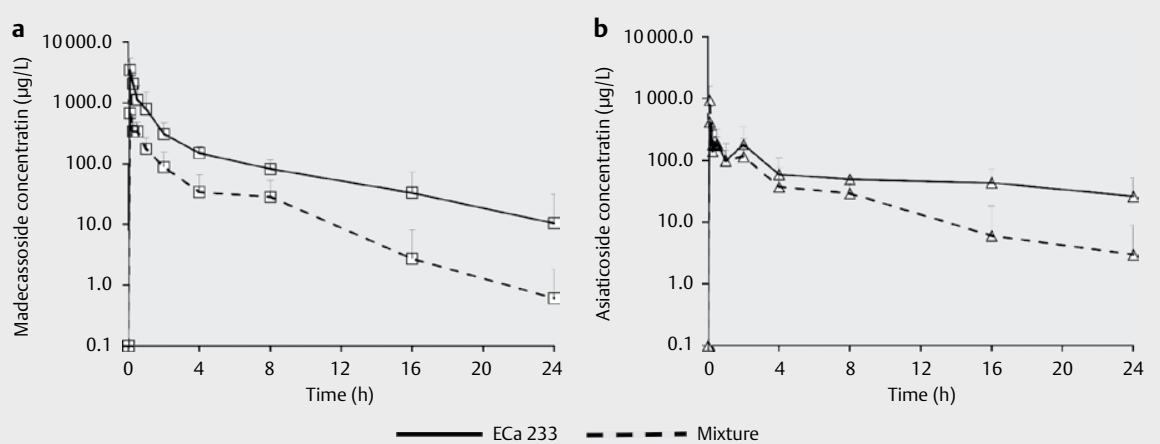

Fig. 3 Plasma concentration-time profiles of madecassoside (a) and asiaticoside (b) following oral administration of ECa 233 (100 mg/kg) and a mixture of madecassoside and asiaticoside at an equivalent dose. Data are presented as the mean \pm S.D. $(n=4) ;{ }^{*} p<0.05$ for ECa 233 vs. mixture.

- Table 2 Pharmacokinetic parameters of madecassoside and asiaticoside after intravenous administration of ECa $23310 \mathrm{mg} / \mathrm{kg}$ and a mixture of madecassoside and asiaticoside at equivalent doses.

\begin{tabular}{|l|c|c|c|c|}
\hline \multirow{2}{*}{$\begin{array}{l}\text { Pharmacokinetic } \\
\text { parameters }\end{array}$} & \multicolumn{2}{|c|}{ Madecassoside } & \multicolumn{2}{c|}{ Asiaticoside } \\
\cline { 2 - 5 } & ECa 233 10 mg/kg & Mixture MD + AS & ECa 233 10 mg/kg & Mixture MD + AS \\
\hline $\mathrm{AUC}_{0-24}(\mu \mathrm{g} . \mathrm{h} / \mathrm{L})$ & $86571.91 \pm 40136.51$ & $22034.21 \pm 7637.14^{*}$ & $20139.85 \pm 7173.22$ & $14707.54 \pm 18920.30^{*}$ \\
\hline $\mathrm{AUC}_{0 \text {-inf }}(\mu \mathrm{g} \cdot \mathrm{h} / \mathrm{L})$ & $88907.03 \pm 41437.76$ & $22362.07 \pm 7655.40^{*}$ & $22558.42 \pm 7997.22$ & $15174.02 \pm 18848.86^{*}$ \\
\hline Vapp $(\mathrm{L} / \mathrm{kg})$ & $0.55 \pm 0.13$ & $2.27 \pm 0.97^{*}$ & $1.99 \pm 0.72$ & $4.45 \pm 3.26$ \\
\hline $\mathrm{MRT}(\mathrm{h})$ & $3.00 \pm 1.42$ & $1.19 \pm 1.07^{*}$ & $7.29 \pm 5.20$ & $3.41 \pm 4.33$ \\
\hline Elimination half-life (h) & $6.21 \pm 1.47$ & $6.14 \pm 2.58$ & $7.80 \pm 3.08$ & $5.59 \pm 2.48$ \\
\hline CLapp $(\mathrm{L} / \mathrm{hr} / \mathrm{kg})$ & $0.07 \pm 0.02$ & $0.25 \pm 0.07$ & $0.19 \pm 0.08$ & $0.52 \pm 0.29$ \\
\hline \multicolumn{2}{|l}{ Data are presented as the mean \pm S.D. $(\mathrm{n}=4) ;{ }^{*} \mathrm{p}<0.05$ for ECa 233 vs. mixture. AS: asiaticoside, MD: madecassoside } \\
\hline
\end{tabular}

\section{Discussion}

Unlike other commercially available extracts of C. asiatica, ECa 233 is a white to off-white natural extract of $C$. asiatica, with known and standardized consistent amounts of its bioactive markers [4]. In agreement with traditional uses, ECa 233 exerts numerous pharmacological activities with minimal toxicity $[6-9,16]$. Some pharmacokinetic studies of ECa 233 at its effective dose range have been conducted in recent years [12]. In the present study, comparative pharmacokinetics between ECa 233 and a mixture of madecassoside and asiaticoside at equivalent amounts were conducted in male Wistar rats. All animals showed good tolerability following IV or PO dosing of the test formulae. No significant changes were observed in physical appearance or kidney and liver markers, which indicates a good safety profile of all test formulae. The results of the present study corresponded well with previous pharmacokinetic studies and toxicity tests of ECa 233 [10].

As shown in the plasma concentration-time profiles, all rats in the ECa 233 group demonstrated significantly higher levels of madecassoside in absorption, distribution, and elimination phases compared with those of the mixture group. Similar response pro- 
- Table 3 Pharmacokinetic parameters of madecassoside and asiaticoside after oral administration of ECa $233100 \mathrm{mg} / \mathrm{kg}$ and a mixture of madecassoside and asiaticoside at equivalent doses.

\begin{tabular}{|c|c|c|c|c|}
\hline \multirow{2}{*}{$\begin{array}{l}\text { Pharmacokinetic } \\
\text { parameters }\end{array}$} & \multicolumn{2}{|c|}{ Madecassoside } & \multicolumn{2}{|c|}{ Asiaticoside } \\
\hline & ECa $233100 \mathrm{mg} / \mathrm{kg}$ & Mixture MD + AS & ECa $233100 \mathrm{mg} / \mathrm{kg}$ & Mixture MD + AS \\
\hline$C_{\max }(\mu \mathrm{g} / \mathrm{L})$ & $3474.63 \pm 1969.04$ & $618.99 \pm 307.64^{*}$ & $509.05 \pm 213.46$ & $697.95 \pm 445.78$ \\
\hline $\mathrm{T}_{\max }(\mathrm{h})$ & $0.08 \pm 0.00$ & $0.19 \pm 0.20$ & $0.19 \pm 0.21$ & $0.08 \pm 0.00$ \\
\hline $\mathrm{AUC}_{0-24}(\mu \mathrm{g} \cdot \mathrm{h} / \mathrm{L})$ & $3,572.14 \pm 1,001.28$ & $747.37 \pm 270.94^{*}$ & $1409.40 \pm 652.63$ & $749.35 \pm 331.33^{*}$ \\
\hline $\mathrm{AUC}_{\mathrm{o}-\text { inf }}(\mu \mathrm{g} \cdot \mathrm{h} / \mathrm{L})$ & $4241.76 \pm 1005.12$ & $1131.52 \pm 840.01^{*}$ & $1787.54 \pm 737.60$ & $888.50 \pm 438.71^{*}$ \\
\hline MRT (h) & $8.52 \pm 3.21$ & $7.21 \pm 6.77$ & $12.72 \pm 4.04$ & $6.48 \pm 4.17$ \\
\hline Elimination half-life (h) & $7.80 \pm 2.24$ & $6.77 \pm 4.99$ & $7.13 \pm 1.75$ & $5.07 \pm 2.01$ \\
\hline
\end{tabular}
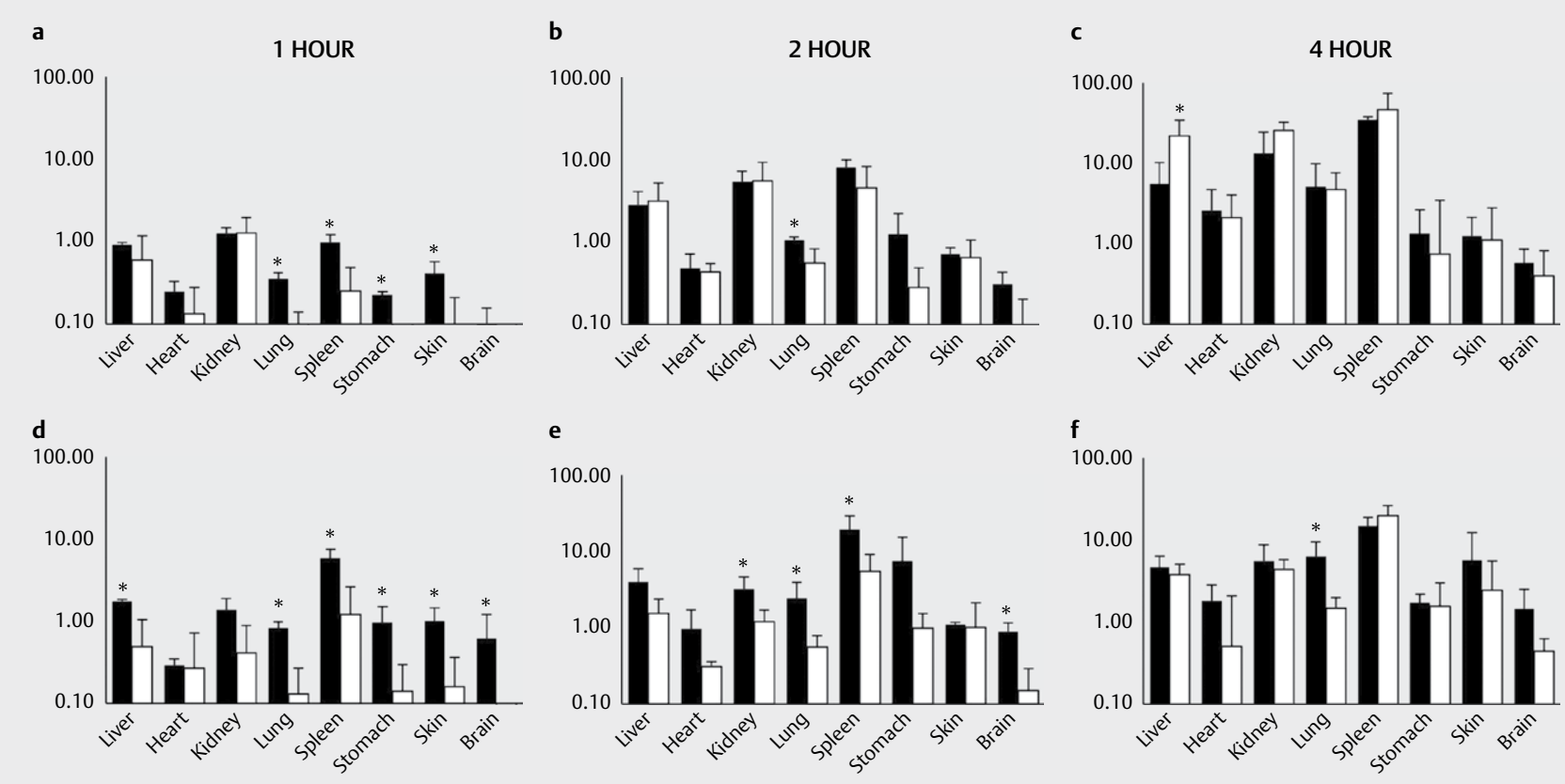

e

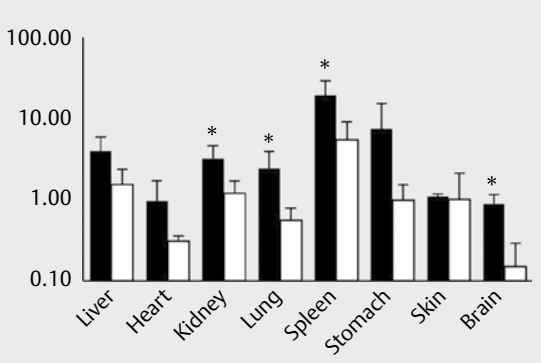

f

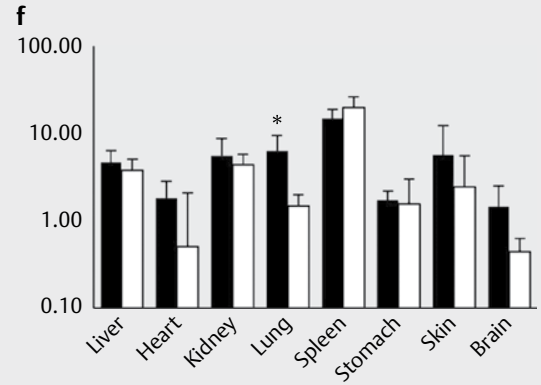

ECa $233 \square$ Mixture

- Fig. 4 Tissue to plasma ratio of madecassoside $(\mathbf{a}, \mathbf{b}, \mathbf{c})$ and asiaticoside $(\mathbf{d}, \mathbf{e}, \mathbf{f})$ in internal organs at 1,2 , and $4 \mathrm{~h}$ after intravenous administration of ECa $233(10 \mathrm{mg} / \mathrm{kg})$ and a mixture of madecassoside and asiaticoside at an equivalent dose. Data are presented as the mean \pm S.D. $(n=4)$.

files were also exhibited by asiaticoside in the ECa 233-treated group where prolonged plasma levels, especially during the elimination phase, were observed. These results were similar to those reported by Hengjumrut et al. [13] in which longer exposure of madecassoside and asiaticoside was observed following administration of ECa 233 compared with the single compounds administered separately. Oral bioavailability of both madecassoside and asiaticoside was increased approximately 2- to 4-fold following administration in the form of ECa 233, suggesting that other minor components present in ECa 233 might play a substantial role as a bioenhancer of madecassoside and asiaticoside [17-19]. Therefore, increases of madecassoside and asiaticoside levels following dosing with ECa 233 were observed from the absorption through the elimination phase.

Determination of the minor components in ECa 233 was conducted using high-resolution LC-MS/MS analysis. It appears likely that ECa 233 contains a certain amount of centellasaponin(s) and is known to contain a small amount of triterpenic acids. In general, appropriate concentrations of minor components to act as bioenhancers should constitute $10 \%$ of the major components. Therefore, it is possible that centellasaponin(s) could act as bioenhancers of the two triterpenoid glycosides in ECa 233. Centellasaponins have structures similar to madecassoside and asiaticoside, which consist of pentacyclic triterpenes and glucose-glucose-rhamnose. These saponins in ECa 233 might have competitive or inhibitory activities toward efflux transporters of madecassoside and asiaticoside. Therefore, improvement of the pharmacokinetic profiles of the two major bioactive components in ECa 233 was observed from the absorption, distribution, and elimination phases. The MRTs of both madecassoside and asiaticoside were improved by intravenous or oral administration of ECa 233 compared with the mixture of pure madecassoside and asiaticoside. Further studies of the bioenhancer activity of centellasaponins could be developed if pure chemicals become commercially available in the future. 


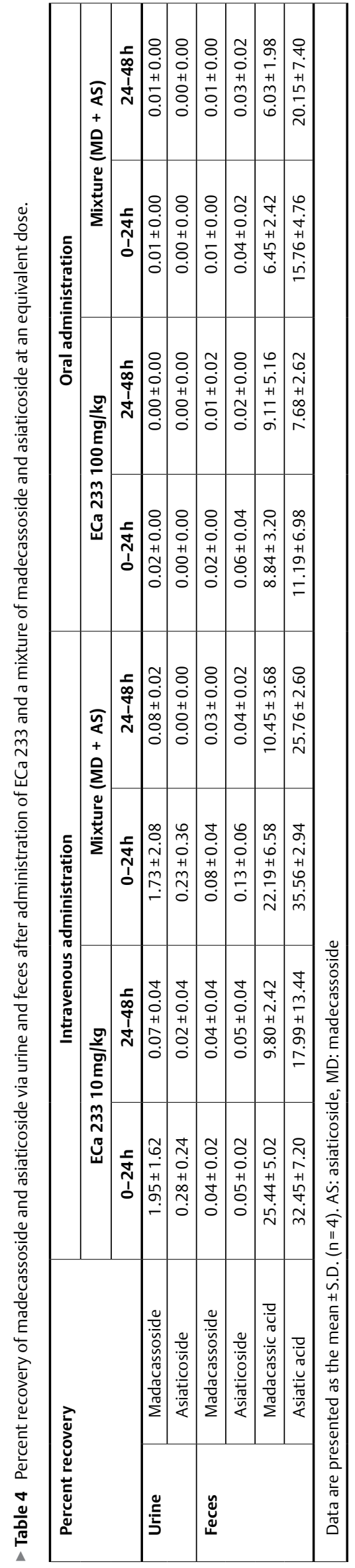

The Kp of both madecassoside and asiaticoside increased over time from 1 to $4 \mathrm{~h}$ following IV dosing of both test formulae. This result correlated well with our previous finding that continuous dosing of $100 \mathrm{mg} / \mathrm{kg}$ PO of ECa 233 for 7 days could significantly increase tissue levels of both triterpenoid glycosides [11]. Madecassoside and asiaticoside are large hydrophilic molecules with molecular weights of more than $900 \mathrm{Da}$. These large triterpenoid molecules might have limited channels to enter tissue compartments compared with small lipophilic molecules. In general, small lipophilic molecules enter tissues by simple diffusion, depending on the concentration gradient of the diffusing molecules [20]. It is likely that the distribution patterns of both triterpenoid glycosides are time dependent rather than concentration dependent. Madecassoside and asiaticoside were able to reach the brain and skin, two major targeted organs of pharmacological activities. The Kp values in these organs increased from 1 to $4 \mathrm{~h}$ following IV dosing of both test formulae, suggesting the potential for ECa 233 to be used as a neuroprotective substance for brain injuries.

In the present study, madecassic and asiatic acids, which have previously been proposed to be the active metabolites [15], were not detected either in plasma or tissue compartments. Therefore, it is likely that administered madecassoside and asiaticoside were absorbed and distributed as unchanged triterpenoid glycosides, not triterpenic acids. Subsequently, the triterpenoid glycosides in the systemic circulation were excreted into the gut lumen via the hepatobiliary system [21]. From there, the unchanged madecassoside and asiaticoside were further biotransformed by the gut flora into triterpenic acids before being excreted in feces $[14,15]$. We found abundant amounts of the two triterpenic acid metabolites, madecassic and asiatic acids, in feces; the percent of recovery was greater than $50 \%$ of the administered doses of triterpenoid glycosides. These two triterpenic acids had very high lipophilic properties, with XlogP ranging from 4.4 to 5.7. Therefore, excretion of these lipophilic molecules should occur via the biliary system rather than the urinary system. P-glycoprotein and multidrug resistance-associated protein 2 are two major transporters responsible for the excretion of madecassoside and asiaticoside into the biliary system [21]. Further information on the hepatobiliary transport and biotransformation of the two triterpenoid glycosides in the gastrointestinal tract will be essential for the development of phytopharmaceutical products from C. asiatica.

Overall, ECa 233, the standardized extract of $C$. asiatica containing mainly madecassoside and asiaticoside, demonstrated superior pharmacokinetic profiles compared with the mixture of madecassoside and asiaticoside at an amount equivalent to their counterparts presented in ECa 233. Higher oral bioavailability and more rapid tissue distribution of triterpenoid glycosides were observed in rats treated with ECa 233. There were no significant differences between the metabolism and excretion profiles of the two triterpenoid glycosides in ECa 233 and the mixture. Pharmacokinetic information obtained from this study clearly demonstrated prolonged exposure of the two major bioactive substances, madecassoside and asiaticoside, of the standardized extract of $C$. asiatica ECa233 compared with a mixture of the two pure compounds. The significant role of the minor constituents on the pharmacokinetic profiles of the major constituents calls for precaution in the interpretation and extrapolation of the data of different extracts. 

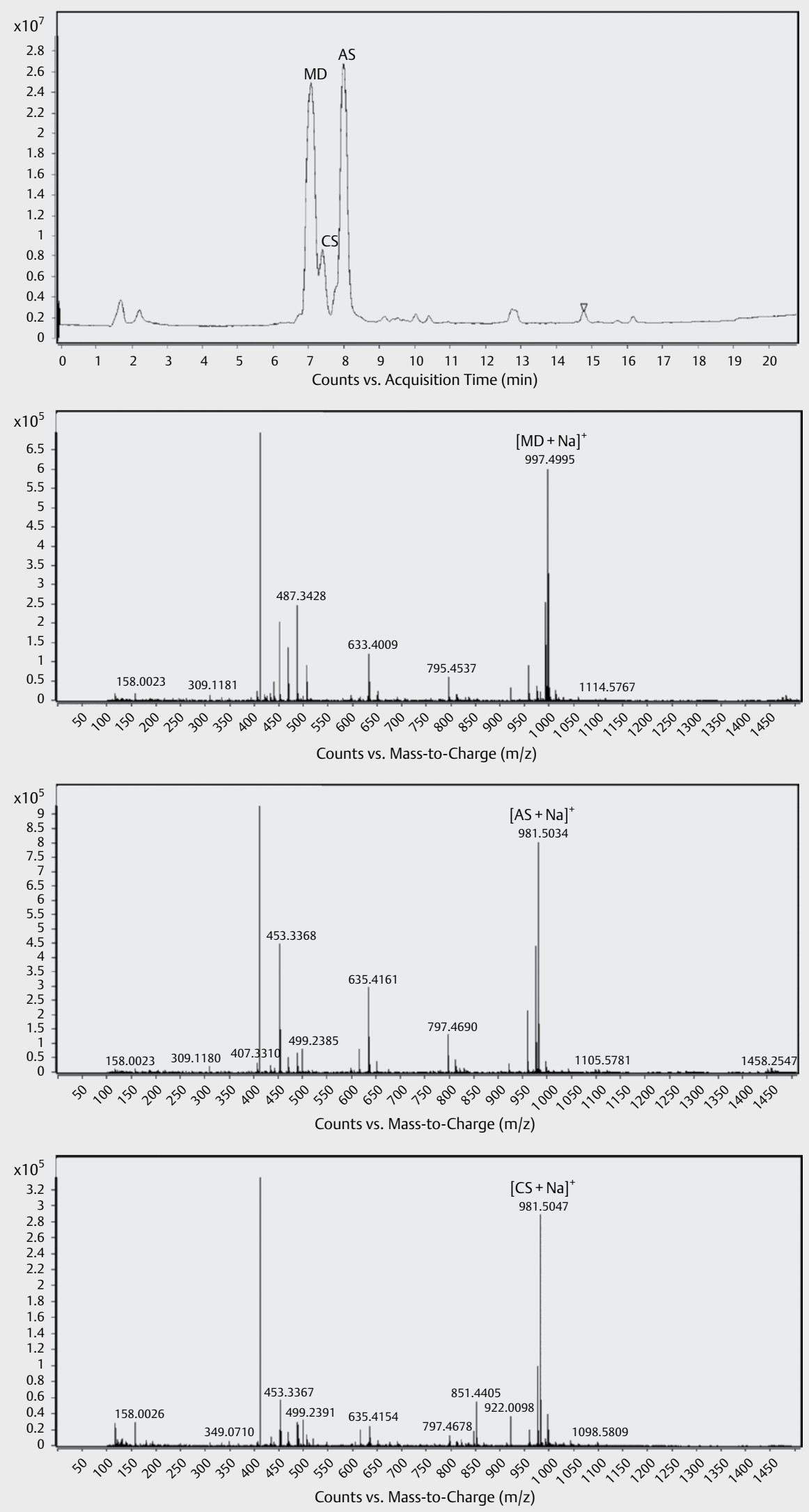

- Fig. 5 MS chromatogram and spectra from high-resolution LC-MS/MS identifying major and minor components of ECa 233. 


\section{Materials and Methods}

\section{Chemicals}

ECa 233, madecassoside, and asiaticoside were provided by Siam Herbal Innovation Co., Ltd. The standardized extract (batch number MRA1214004) contained $51 \%$ madecassoside and 38\% asiaticoside, and was analyzed by LC-MS/MS. Analytical standards of asiaticoside ( $>98.5 \%$ ), madecassoside ( $>95.0 \%$ ), and asiatic acid ( $>95.0 \%$ ) were purchased from Sigma-Aldrich Corp. Madecassic acid (>98.9\%) and glycyrrhetinic acid (>99.0\%) used for LC-MS/MS experiments were purchased from Wako Pure Chemical Industries.

\section{Animals and treatments}

Male Wistar rats were obtained from the National Laboratory Animal Center, Mahidol University, Thailand. The rats were housed under 12 -h light-dark cycles, $24 \pm 2{ }^{\circ} \mathrm{C}, 50 \pm 10 \%$ humidity, and ad libitum access to food and water. Rats aged 16-20 weeks that weighed more than $400 \mathrm{~g}$ were used in the pharmacokinetic experiments; they were placed into metabolic cages and fasted overnight with free access to water. The animal protocols were approved by the Institutional Animal Care and Use Committee of the Faculty of Pharmaceutical Sciences, Chulalongkorn University, Thailand (approval number 15-33-002, approval date April 22, 2015).

\section{Pharmacokinetic experiments}

The rats were divided into four groups $(n=4)$ : ECa $23310 \mathrm{mg} / \mathrm{kg}$ IV, ECa $233100 \mathrm{mg} / \mathrm{kg}$ PO, a mixture of madecassoside and asiaticoside (5.1 and $3.8 \mathrm{mg} / \mathrm{kg}$, respectively) IV, and a mixture of madecassoside and asiaticoside (51 and $38 \mathrm{mg} / \mathrm{kg}$, respectively) PO. All formulations were freshly prepared as a clear solution in $20 \%$ DMSO/NSS before administration via the lateral tail vein or oral gavage. The rats were anesthetized with isoflurane during drug administration and blood or organ collections. We collected $300 \mu \mathrm{L}$ of blood via the lateral tail vein at $0,5,15$, and $30 \mathrm{~min}$, and 1, 2, 4, 8, 16 , and $24 \mathrm{~h}$ after dosing. Blood samples were collected from these rats and centrifuged at $1500 \times \mathrm{g}$ for $15 \mathrm{~min}$ to collect $150 \mu \mathrm{L}$ of plasma. Plasma samples at 0 and $24 \mathrm{~h}$ were used to determine creatinine, AST, and ALT levels. Tissue samples were collected at 1, 2, and $4 \mathrm{~h}$ after IV dosing, and washed with cold saline solution. Rat excreta, urine, and feces were collected separately from the metabolic cages $24-48 \mathrm{~h}$ after dosing to determine the excretion routes of drugs and metabolites. All biological samples were stored at $-80^{\circ} \mathrm{C}$ until analysis.

\section{Sample preparation}

Methanol was used as a precipitating agent in the protein precipitation method. Fifty microliters of plasma or urine samples were thawed at room temperature and vigorously mixed with $200 \mu \mathrm{L}$ of methanol containing $10 \mathrm{ng}$ of glycyrrhetinic acid as the IS. The mixture was centrifuged at $3000 \times \mathrm{g}$ for $15 \mathrm{~min}$, and the supernatants $(10 \mu \mathrm{L})$ were analyzed by LC-MS/MS. Fifty milligrams of feces or tissues were mixed with $200 \mu \mathrm{L}$ of methanol that contained $10 \mathrm{ng}$ of IS, homogenized in an ice bath, and processed in the same manner as the liquid samples. In the case that the drug or metabolite levels exceeded the linearity of the calibration curves, blank matrices were used to dilute the samples prior to protein precipitation.

\section{LC-MS/MS analysis}

LC-MS/MS analysis was conducted under conditions and methods previously described in a pharmacokinetic study of ECa 233 [12]. In brief, an LC-MS/MS system was conducted with an Eksigent UPLC 100 and a QTRAP 6500 mass spectrometer and Analyst software version 1.6 (AB Sciex Pte., Ltd.). The stationary phase was a Synergi Fusion-RP C18 column (Phenomenex, Inc.) with a $40^{\circ} \mathrm{C}$ oven temperature. The retention times of madecassoside, asiaticoside, madecassic acid, asiatic acid, and glycyrrhetinic acid were 1.79, 1.82, $1.93,1.99$, and $2.12 \mathrm{~min}$, respectively. The standard curves of the triterpenoid glycosides and triterpenic acids showed good linearity, with $R^{2}>0.99$ for $0.5-300 \mu \mathrm{g} / \mathrm{L}$. The lower limit of detection was $0.1-0.5 \mu \mathrm{g} / \mathrm{L}$, and intra- and inter-day precision and accuracy were within $\pm 10 \%$. The calculated percent recoveries were more than $70 \%$ for all analytes.

\section{Minor components identification}

In this study, the identification of the minor components of ECa 233 was conducted with high-resolution LC-MS/MS analysis. LC-MS/MS analysis was performed on an Agilent 6530 QTOF mass spectrometer with an Agilent HPLC 1260 binary pump. A Kinetex Phenomenex $C_{18}$ column $(3.5 \mu \mathrm{m}, 2.1 \times 150 \mathrm{~mm})$ was used as the stationary phase. The mobile phase was run with a gradient of $0.1 \%$ formic acid in water and acetonitrile (20-80\%) at a flow rate of $0.2 \mathrm{~mL} /$ min. The mass spectrometer was equipped with an Agilent Jet Stream ESI source. LC-MS/MS was performed in the positive ionization mode over a mass range of $\mathrm{m} / \mathrm{z} 100-1700$ at a scan rate of 3 spectra/second. ECa $233(10 \mu \mathrm{L})$ at a concentration of $0.1 \mathrm{mg} / \mathrm{mL}$ was injected into the $\mathrm{LC}$ system, with the column oven maintained at $25{ }^{\circ} \mathrm{C}$. The elution gradient of the mobile phase started with $20 \%$ acetonitrile at time zero and increased to $80 \%$ acetonitrile after $20 \mathrm{~min}$, was maintained at $80 \%$ acetonitrile for $3 \mathrm{~min}$, decreased to $20 \%$ acetonitrile over $5 \mathrm{~min}$, and was equilibrated with $20 \%$ acetonitrile for $5 \mathrm{~min}$. Analysis of the eluates was conducted in the positive ion mode of ionization, and employed a QTOF mass spectrometer (QTOF 6530, Agilent Technologies). Qualitative analysis of mass spectra was performed with MassHunter software B.07.00 (Agilent Technologies).

\section{Data analysis}

The pharmacokinetic parameters of madecassoside and asiaticoside were calculated by non-compartmental analysis using PK solution software (Summit Research Service). The $C_{\max }$ and $T_{\max }$ were directly observed from real experimental data. The $\mathrm{AUC}_{0-24}, \mathrm{AUC}_{0-\mathrm{inf}}, \mathrm{V}_{\mathrm{app}}$, $\mathrm{T}_{1 / 2}, \mathrm{Cl}_{\mathrm{app}}$, and MRT were reported. The Kp values were calculated from the drug concentrations in the tissue divided by the drug concentration in the plasma at the same time point. The percent of recovery of the drugs and metabolites in the excreta was calculated as the total drugs or metabolites found in the excreta divided by the administered dose based on molarity. All parameters were reported as the mean \pm standard deviation. Statistical differences in pharmacokinetic parameters between the two experimental groups were analyzed using a nonparametric test $(p<0.05)$. SPSS statistical analysis (version 16) was used for all data analyses (SPSS, Inc.). 


\section{Supporting information}

A representative LC-MS/MS chromatogram from a plasma sample and MS/MS fragments of major and minor components of ECa 233 are available as Supporting Information.

\section{Acknowledgments}

All authors would like to thank Dr. Mengke Zhang and Mr. Justin Reinicke for their kind support and assistance in high-resolution LC-MS/MS analysis at the Daniel K. Inouye College of Pharmacy, University of Hawai'i at Hilo. The authors are grateful for the overseas research experience and scholarship of Chulalongkorn University (to Phisit Khemawoot). We also express our gratitude to the Faculty of Pharmaceutical Sciences, Chulalongkorn University for providing research funds for the study (Grant number, Phar2561RG002).

\section{Conflict of Interest}

All authors declare no conflicts of interest.

\section{References}

[1] Bown D. Encyclopedia of Herbs and Their Uses. New York: Dorling Kindersley; 1995

[2] Jamil SS, Nizami Q, Salam M. Centella asiatica (Linn.) Urban: A review. Indian J Nat Prod Res 2007; 6: 158-170

[3] Krittapun W. Usefulness of herbs. Salaya, Thailand: Department of Pharmacognosy, Faculty of Pharmacy, Mahidol University; 1996

[4] Saifah E, Suttisri R, Patarapanich C, Laungchonlatan S, Tantisira MH, Tantisira B. Preparation methods of colorless mixture between madecassoside and asiaticoside from Centella asiatica. In: Department of Intellectual Property MoC ed. C07H 1/00 ed. Bangkok, Thailand: Chulalongkorn University; 2009

[5] Tantisira MH. Bioactive standardized extract of Centella asiatica (ECa 233).In: The Eighth NRCT-JSPS Joint Seminar. Bangkok, Thailand: Chulalongkorn University Printing Press; 2009: 25

[6] Kam-eg A, Tantisira B, Tantisira MH. Preliminary study on effects of a standardized extract of Centella asiatica, ECa 233, on deficit of learning and memory induced by an intracerebroventricular injection of beta-amyloid peptide in mice. Thai J Pharmacol 2009; 31: 79-82

[7] Ruengprasertkit C, Hongprasong N, Tantisira B, Tantisira MH. Preliminary study of effects of a standardized extract of Centella asiatica ECa 233 on minor aphthous ulcers. Chulalongkorn Univ Dent J 2010; 33: 131-142
[8] Wanakhachornkrai O, Pongrakhananon V, Chunhacha P, Wanasuntronwong A, Vattanajun A, Tantisira B, Chanvorachote P, Tantisira M. Neuritogenic effect of standardized extract of Centella asiatica ECa 233 on human neuroblastoma cells. BMC Complement Altern Med 2013; 13: 204

[9] Wannarat K, Tantisira MH, Tantisira B. Wound healing effects of a standardized extract of Centella asiatica ECa 233 on burn wound in rats. Thai J Pharmacol 2009; 31: 120-123

[10] Chivapat S, Chavalittumrong P, Tantisira MH. Acute and sub-chronic toxicity studies of a standardized extract of Centella asiatica ECa 233. Thai J Pharmaceut Sci 2011; 35: 55-64

[11] Anukunwithaya T, Tantisira MH, Shimada T, Sai Y, Khemawoot P. Multiple oral dosing pharmacokinetics of standardized extract of Centella asiatica ECa 233 and its inductive effect on efflux transporters in rats. PMIO 2017; 4: e66-e73

[12] Anukunwithaya T, Tantisira MH, Tantisira B, Khemawoot P. Pharmacokinetics of a standardized extract of Centella asiatica ECa 233 in rats. Planta Med 2017; 83: 710-717

[13] Hengjumrut P, Anukunwithaya T, Tantisira MH, Tantisira B, Khemawoot P. Comparative pharmacokinetics between madecassoside and asiaticoside presented in a standardised extract of Centella asiatica, ECa 233 and their respective pure compound given separately in rats. Xenobiotica 2018; 48: 18-27

[14] Bossé JP, Papillon J, Frenette G, Dansereau J, Cadotte M, Le Lorier J. Clinical study of a new antikeloid agent. Ann Plast Surg 1979; 3: 13-21

[15] Chassaud LF, Fry BJ, Hawkins DR, Lewis JD, Sword IP, Taylor T, Hathway DE. The metabolism of asiatic acid,-madecassic acid and asiaticoside in the rat. Arzneimittelforschung 1971; 21: 1379-1384

[16] Wanasuntronwong A, Tantisira MH, Tantisira B, Watanabe H. Anxiolytic effects of standardized extract of Centella asiatica (ECa 233) after chronic immobilization stress in mice. J Ethnopharmacol 2012; 143: 579-585

[17] Kesarwani K, Gupta R, Mukerjee A. Bioavailability enhancers of herbal origin: an overview. Asian Pac J Trop Biomed 2013; 3: 253-266

[18] Ajazuddin Alexander A, Qureshi A, Kumari L, Vaishnav P, Sharma M, Saraf S, Saraf S. Role of herbal bioactives as a potential bioavailability enhancer for Active Pharmaceutical Ingredients. Fitoterapia 2014; 97 : $1-14$

[19] Dudhatra GB, Mody SK, Awale MM, Patel HB, Modi CM, Kumar A, Kamani DR, Chauhan BN. A comprehensive review on pharmacotherapeutics of herbal bioenhancers. ScientificWorldjournal 2012; 2012: 637953

[20] Yang NJ, Hinner M]. getting across the cell membrane: an overview for small molecules, peptides, and proteins. In: Gautier A, Hinner MJ. editors. Site-specific protein labeling: Methods and protocols. New York, NY: Springer New York; 2015: 29-53

[21] Leng DD, Han W], Rui Y, Dai Y, Xia YF. In vivo disposition and metabolism of madecassoside, a major bioactive constituent in Centella asiatica (L.) Urb. J Ethnopharmacol 2013; 150: 601-608 